\title{
Correlation and multiplicity measurements from RHIC to the LHC
}

\section{Gábor I. Veres* 'Eötvös Loránd University, Budapest, Hungary}

E-mail: gabor.veres@cern.ch

\begin{abstract}
A selection of experimental results and methods is reviewed in connection to the investigation of heavy ion collisions using correlations involving hard probes and related bulk quantities. Correlations between high transverse momentum trigger particles and the charged hadrons created in soft processes in the same collision can reveal interesting aspects of the interaction between the hard probe and the medium. It is argued that understanding the general features of the global event characteristics like particle density and elliptic flow, in addition to the availability of a large geometrical detector coverage, are essential to the interpretation of those results. In this context, some of the universal scaling features in the heavy ion data collected at RHIC, together with expectations for the LHC, will be presented. Besides those predictions, preparations for measurements of particle distributions and elliptic flow at the CMS experiment at the LHC will be discussed. The review will conclude with a detailed study on high- $p_{T} \gamma$-jet correlations providing the possibility to measure jet fragmentation functions, relying on the large coverage CMS electromagnetic calorimeters.
\end{abstract}

4th international workshop High-pT physics at LHC 09

February 4-7, 2009

Prague, Czech Republic

\footnotetext{
* Speaker.

${ }^{\dagger}$ Also at CERN - European Organization for Nuclear Research
} 


\section{Introduction}

The general goal of the experimental studies of high energy nuclear collisions is to create a new phase of matter at very high density and temperature, where partons become the relevant degrees of freedom, instead of hadrons. The conditions in the created medium are thought to be similar to that in the early Universe well before the nucleosynthesis. Significant part of the experimental evidence that a new form of matter is created in these collisions comes from results related to high transverse momentum (high- $p_{T}$ ) particles. At the LHC, fully developed jets will also be reconstructed. The interaction between those high- $p_{T}$ objects and the produced medium is considered to be a useful tool to reveal certain features of the elusive new form of matter. Correlation measurements between high- $p_{T}$ particles and the hadrons constituting the 'bulk' of the heavy ion events have gained a lot of importance recently [1,2].

These correlation measurements are not independent of the general bulk features of the heavy ion event. In particular, elliptic flow correlations form an important 'background' to the 'jet-bulk' correlations. Experimental biases caused by selecting a high- $p_{T}$ particle (or jet) in the event depend on the pseudorapidity-density, $d N / d \eta$. Particle yields per trigger particle are also calculated from the correlation functions using the inclusive $d N / d \eta$ distributions [3, 4].

Concerning the interpretation of the correlation measurements, a lot can be learned from extending the $\eta$-region (or $\Delta \eta$ region) of these studies beyond mid-rapidity [5, 6]. However, the above mentioned bulk quantities strongly depend on the pseudo-rapidity, $\eta$. The dependence is not an arbitrary one, on the contrary: various simple regularities, scaling and factorization features can be observed in the data taken at different collision energies, nuclear sizes and centralities [7]. Some of these important observations will be mentioned, the understanding of which are probably necessary to complete our physical picture of the correlations found in the data.

It will be important to achieve a good assessment of these bulk features at the LHC as well, in particular to be able to account for backgrounds and biases in the correlation measurements with high- $p_{T}$ probes. The preparations within the CMS heavy ion program for the measurement of $d N / d \eta$ and elliptic flow $\left(v_{2}\right)$ will be discussed, together with the capabilities of CMS to measure various types of correlations. As an important example, a Monte-Carlo simulation based analysis of gamma-jet correlations will be presented, as a means of determining the quenching effect on the jet fragmentation functions, utilizing the large acceptance and high segmentation of the CMS electromagnetic calorimeters.

\section{Correlations with a high- $p_{T}$ particle}

One of the most important discoveries of the Relativistic Heavy Ion Collider (RHIC) is that high-energy partons interact strongly and lose a large fraction of their energy as they traverse the medium produced in heavy ion collisions. The amount of energy loss can be estimated already from charged hadron transverse momentum spectra in nuclear collisions, compared to more elementary (usually $\mathrm{p}+\mathrm{p}$ ) collisions $[8,9,10,11]$. A widely used method to quantify the suppression of high- $p_{T}$ particle production in heavy ion collisions is to calculate the nuclear modification factors, defined as:

$$
R_{A A}\left(p_{T}\right)=\frac{\sigma_{p p}^{\text {inel }}}{\left\langle N_{\text {coll }}\right\rangle} \frac{d^{2} N_{A A} / d p_{T} d \eta}{d^{2} \sigma_{p p} / d p_{T} d \eta}
$$



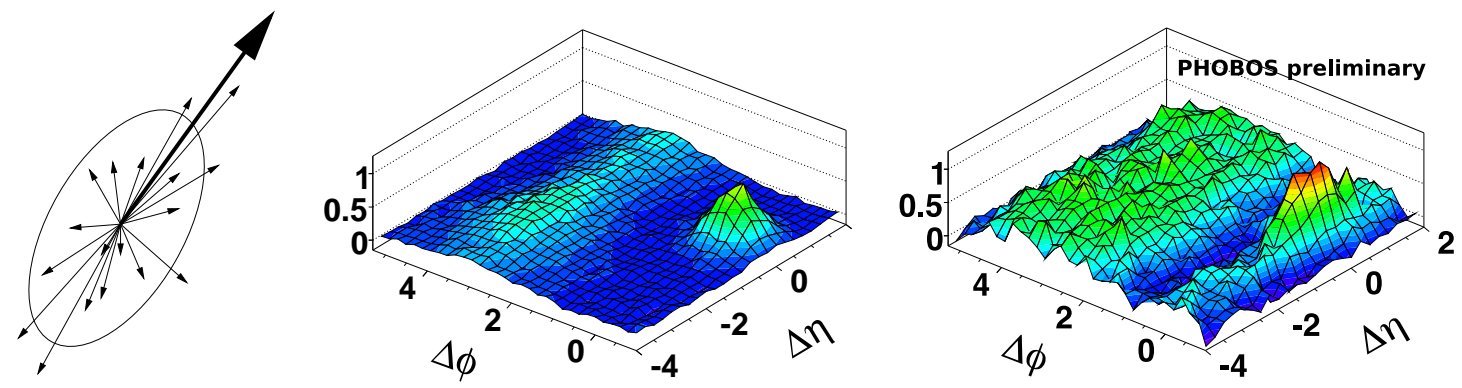

Figure 1: Left: the sketch of the two particle azimuthal angle correlation measurement. The thick arrow represents the high- $p_{T}$ trigger particle, while the other arrows depict associated particles, in a plane perpendicular to the beam. The ellipse symbolizes the azimuthal angle distribution (averaged over many events). Center: correlated yield per trigger particle, using $p_{T}^{\text {trig }}>2.5 \mathrm{GeV} / \mathrm{c}$, as a function of $\Delta \eta$ and $\Delta \phi$ for $200 \mathrm{GeV} \mathrm{p}+\mathrm{p}$ events generated by PYTHIA [12]. Right: the correlated yield for Au+Au collisions at $200 \mathrm{GeV} /$ nucleon pair collision energy, measured by PHOBOS [3, 4].

where $\left\langle N_{\text {coll }}\right\rangle$ is the mean number of binary nucleon-nucleon collisions in a given centrality class of the data. Without nuclear effects, $R_{A A}=1$ is expected at high $p_{T}$.

The effect of the large amount of energy lost by energetic partons traversing the medium is also observed in azimuthal correlations, where back-to-back high- $p_{T}$ particles disappear in central $\mathrm{Au}+\mathrm{Au}$ collisions [13]. In these measurements, a high- $p_{T}$ trigger particle is selected from a given heavy ion event, and some, or all of the other particles in the same event are called associated particles, as sketched on the left panel of Fig. 1. The correlation between the direction of the trigger and associated particles is measured, usually both in the azimuthal angle $(\phi)$ and pseudorapidity $(\eta)$ direction. For associated particles selected to have high momentum, the behavior is consistent with the surface emission of jets, caused by the presence of an opaque medium that completely absorbs those jets directed at the interior of the collision zone.

The energy and momentum of the away-side jet has to be present in the final state due to the energy-momentum conservation, and it is necessarily carried by the associated particles in the same event. Therefore, there is a strong motivation to study the correlations between high- $p_{T}$ trigger and lower $p_{T}$ associated particles. Close to mid-rapidity, data collected at RHIC show several nontrivial features of those correlation functions. In addition to a broadening of the awayside structure relative to $\mathrm{p}+\mathrm{p}[1,2]$, a clear enhancement in the correlation near $\Delta \phi=0$ is also observed. This structure has been called the 'ridge' [14]. Although the ridge close to mid-rapidity has been qualitatively described by a diverse assortment of proposed mechanisms using various theoretical approaches $[5,15,16,17,18,19,20,21]$, the precise origin of the structure is still not well understood. The PHOBOS collaboration have used the uniquely broad $\eta$ acceptance of their multiplicity detector to measure the ridge structure (and its dependence on event centrality) at large relative pseudo-rapidity $(\Delta \eta)$, and thereby constrain the possible interpretations of particle production correlated with high- $p_{T}$ trigger particles.

The trigger particles used in the PHOBOS data have been selected with $p_{T}>2.5 \mathrm{GeV} / \mathrm{c}$ and $0<\eta^{\text {trig }}<1.5$. Associated particles $\left(p_{T}>4 \mathrm{MeV} / \mathrm{c}\right.$ at $\eta \approx 3, p_{T}>35 \mathrm{MeV} / \mathrm{c}$ at $\eta \approx 0$ ) are detected in a single-layered silicon detector, covering $|\eta|<3$.

The raw per-trigger distribution of same-event pairs, $s(\Delta \phi, \Delta \eta)$ was divided by the raw distri- 

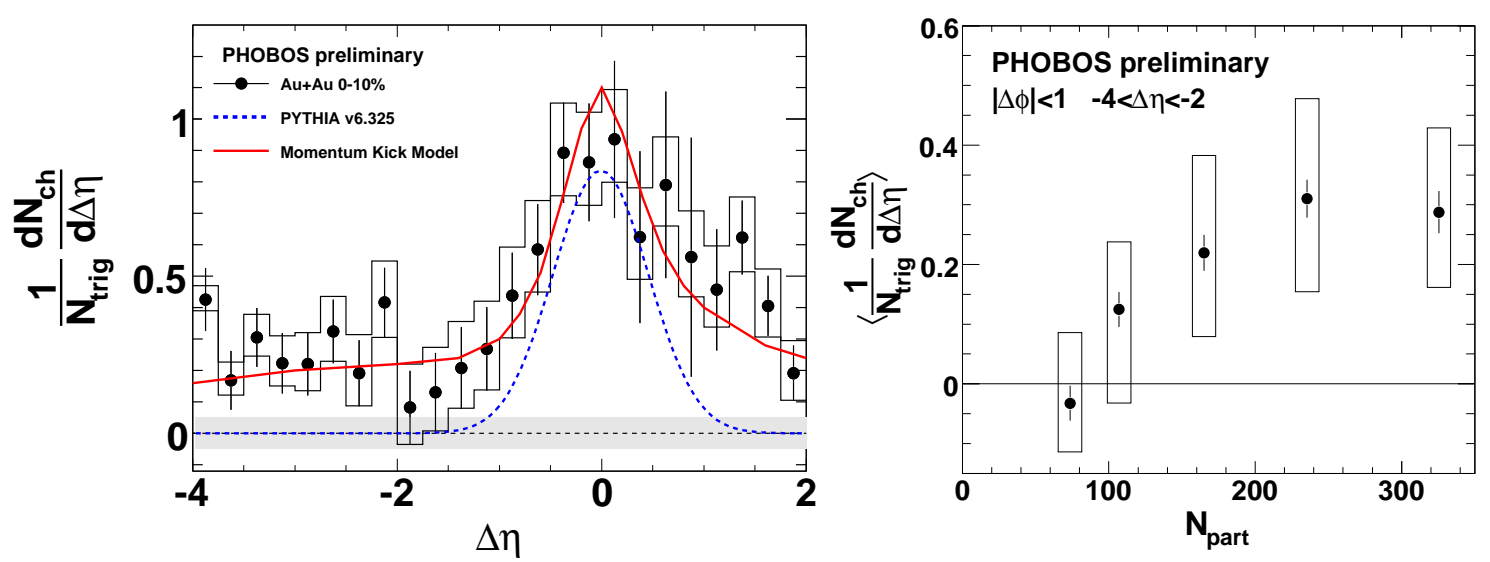

Figure 2: Left: per-trigger correlated yield for central (0-10\%) Au+Au events measured by PHOBOS [3, 4], integrated over the near side $(|\Delta \phi|<1)$ compared to PYTHIA [12] (dashed line) and to the momentum kick model prediction [5] (solid line) as a function of $\Delta \eta$. Boxes represent the systematic uncertainty. Right: average yield of the ridge as a function of $N_{\text {part }}$ in the $-4<\Delta \eta<-2$ range from the left plot [3, 4]. Boxes represent the systematic errors.

bution of mixed-event pairs (the 'background'), $b(\Delta \phi, \Delta \eta)$, to remove detector effects:

$$
\frac{1}{N_{\text {trig }}} \frac{d^{2} N_{c h}}{d \Delta \phi d \Delta \eta}=B(\Delta \eta) \cdot\left[\frac{s(\Delta \phi, \Delta \eta)}{b(\Delta \phi, \Delta \eta)}-a(\Delta \eta)[1+2 V(\Delta \eta) \cos (2 \Delta \phi)]\right]
$$

The last term in Eq. 2.2 is due to elliptic flow, quantified by $v_{2}$. It is completely erased in the event mixed sample, giving a uniform background distribution in $\Delta \phi$. However, both the trigger and the associated particles are affected by $v_{2}$, resulting in a flow modulation, to be subtracted from the correlation. The modulation is denoted by $V(\Delta \eta)$, and can be approximated by $\left\langle v_{2}^{\text {trig }}\right\rangle \times\left\langle v_{2}^{\text {assoc }}\right\rangle$, with each term taken from the published PHOBOS $v_{2}$ results, in a factorized form vs. $N_{\text {part }}, p_{T}$ and $\eta$ [22]. The scale factor $a(\Delta \eta)$ in Eq. 2.2 is introduced to balance the trigger bias between signal and mixed-event distributions, calculated with the ZYAM method [23]. $B(\Delta \eta)$ is the corrected, published inclusive $d N / d \eta$ distribution [24] convoluted with the $\eta$ distribution of trigger particles.

The $\mathrm{Au}+\mathrm{Au}$ correlation from PHOBOS is shown on the right panel of Fig. 1, and can be compared to those in $p+p$ events simulated with PYTHIA [12] (center panel of Fig. 1). The correlation in $\mathrm{p}+\mathrm{p}$ events has a jet-fragmentation peak close to $\Delta \eta \approx \Delta \phi \approx 0$ and an away-side structure around $\Delta \phi \approx \pi$ that has similar width in $\Delta \phi$ but is much more extended in $\Delta \eta$. In central Au+Au collisions, not only is the away-side structure broader in $\Delta \phi$, but the near-side peak now sits on a pedestal, a ridge of correlated partners extending (at least) to $|\Delta \eta|=4[4,25]$.

To further examine the near-side structure, the correlated yield is integrated over $|\Delta \phi|<1$ and plotted as a function of $\Delta \eta$ on the left panel of Fig. 2 for the $10 \%$ most central Au+Au collisions. There is an approximately $\Delta \eta$-independent correlated yield of about 0.3 particles per unit of pseudo-rapidity, even at large $\Delta \eta$. The prediction of the momentum kick model [5] is found to agree well with the data. This model postulates a parton $y$-distribution at the time of the jet-parton collision that is broader than the $y$-distribution of final state hadrons. That suggests that the shape of the ridge is sensitive to the earliest moments of the $\mathrm{Au}+\mathrm{Au}$ collision, and can be used as a probe of the initial parton $y$-distributions [6]. The centrality dependence of the average ridge yield far 

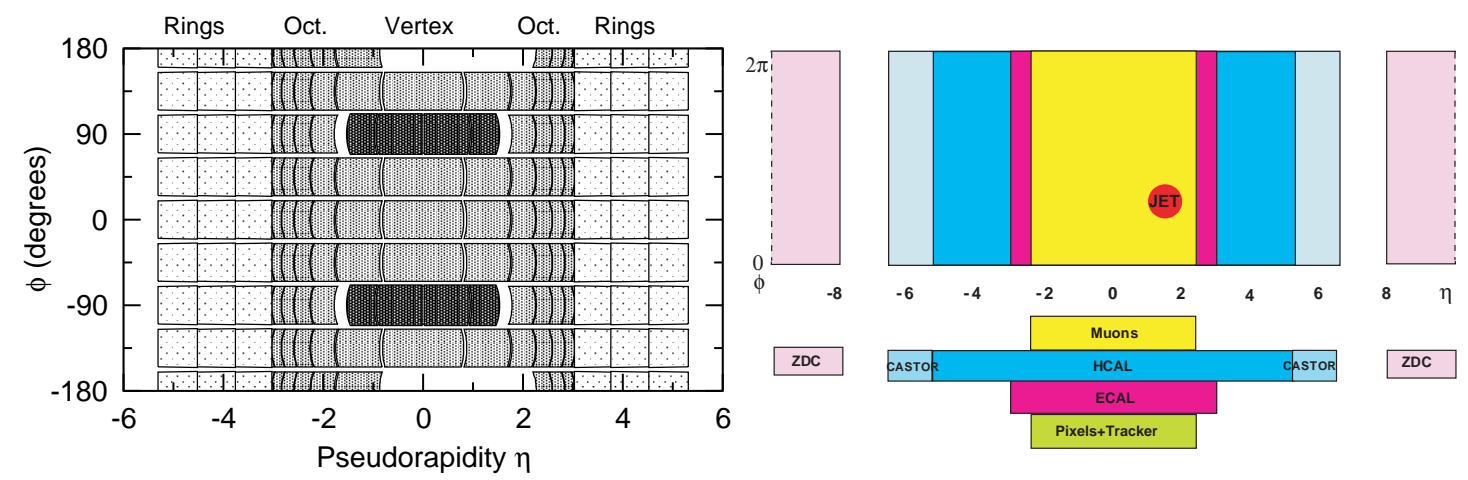

Figure 3: Left: the geometrical acceptance of the PHOBOS Ring (light), Octagon (medium) and Inner Vertex (dark) detectors for particles emitted from the nominal interaction point, as a function of $\eta$ and $\phi$ [26]. Right: CMS acceptance of tracking, calorimetry, and muon identification in $\eta$ and $\phi$. The size of a jet with a cone $\mathrm{R}=0.5$ is depicted for illustration.

away from the trigger $(-4<\Delta \eta<-2)$ is shown on the right panel of Fig. 2. The ridge yield decreases towards more peripheral collisions, until it vanishes and becomes consistent with zero in the most peripheral bin analyzed, at $\left\langle N_{\text {part }}\right\rangle=80[3,4]$.

\section{Correlations and bulk properties}

As discussed in the last section, extending mid-rapidity measurements to large $|y|$ or $|\eta|$ regions is extremely valuable for the interpretation of correlation results and to test phenomenological models. While it has been possible to cover almost the full longitudinal phase phase with active detectors in fixed-target experiments (like e.g. NA49 and NA61 at the SPS), with the increasing collision energy frontier - meaning collider experiments - it is more and more difficult. Examples of collider experiments known for their outstanding forward instrumentation are BRAHMS and PHOBOS at RHIC, and CMS and TOTEM at LHC. For illustration, the $\eta-\phi$ coverage is shown on Fig. 3 for PHOBOS and CMS. The single-layered silicon detector for charged particle detection of PHOBOS is extended to $|\eta|<5$.4. In CMS, full tracking information is available for $|\eta|<2.4$, the electromagnetic $(|\eta|<3)$, and hadronic $(|\eta|<5)$ calorimetry is extended with the CASTOR calorimeter into the $5<|\eta|<7$ and with the ZDC into the $|\eta|>8$ region.

Besides having the experimental capability, the systematic study of the bulk quantities as a function of energy, centrality (system geometry) and system size has found that the data exhibit remarkable scaling and factorization features. Particularly, the $d N / d \eta$ and $v_{2}$ measurements and its features are strongly related to correlation measurements with high- $p_{T}$ trigger particles, as discussed earlier. In this section, some of these scaling features will be presented.

The extended longitudinal scaling of the $d N / d \eta$ distributions in Au+Au collisions measured by the PHOBOS experiment at various collision energies is illustrated on the left panel of Fig. 4 for central collisions. By plotting the particle density as a function of $\eta^{\prime}=\eta \pm y_{\text {beam }}$ (essentially transforming the frame of reference to the rest frame of each projectile), the data fall onto a common, energy-independent limiting curve, in a sizeable $\eta^{\prime}$ region. Assuming this scaling behavior to hold for higher energies, and that the mid-rapidity particle density is the logarithmic function of 

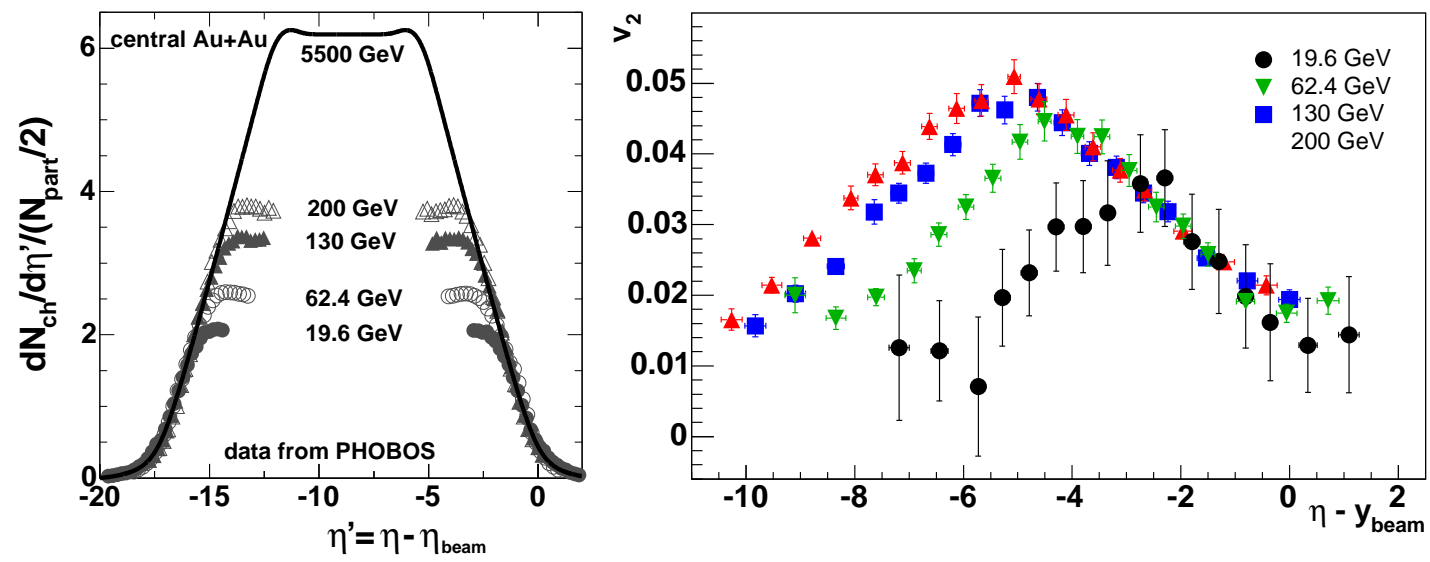

Figure 4: Left: Pseudo-rapidity distributions, $d N_{c h} / d \eta$, normalized by the number of participating nucleon pairs, for primary charged hadrons in central $\mathrm{Au}+\mathrm{Au}$ collisions at RHIC $[27,28]\left(\sqrt{s_{N N}}=19.6-200 \mathrm{GeV}\right)$ and extrapolation for $\mathrm{Pb}+\mathrm{Pb}$ at LHC $\left(\sqrt{s_{N N}}=5500 \mathrm{GeV}\right)$. Right: The elliptic flow measured by PHOBOS, in $\mathrm{Au}+\mathrm{Au}$ collisions at $\sqrt{s_{N N}}=19.6-200 \mathrm{GeV}$, in the $0-40 \%$ centrality range, as a function of $\eta-y_{\text {beam }}$ [29]. The error bars represent the statistical errors $(1 \sigma)$.

$\sqrt{s}$, one obtains a simple data-driven expectation for the $d N / d \eta$ distribution at the LHC (for the nominal collision energy of $5.5 \mathrm{GeV} /$ nucleon pair) [27, 28], as depicted by the solid line on the left panel of Fig. 4.

Another remarkable feature of the bulk quantities is a similar longitudinal scaling of the $v_{2}$ coefficients characterizing the elliptic flow, observed by the PHOBOS experiment. The $v_{2}$ values measured in $\mathrm{Au}+\mathrm{Au}$ collisions at various collision energies, again, as a function of $\eta^{\prime}=\eta-y_{\text {beam }}$, fall onto a common, energy-independent limiting curve [29], as shown on the right panel of Fig. 4. The precise measurements of the longitudinal features of the bulk properties are important for understanding the correlation results. In particular, an extensive data set on $v_{2}$ as a function of centrality, $p_{T}$ and $\eta$ was used to correctly evaluate the associated yield to a high- $p_{T}$ trigger particle at large $\Delta \eta$ values.

The comparison of collisions with different centralities and system sizes (nuclear mass numbers) is useful to study the role of the collision geometry in the $d N / d \eta$ distributions. A recent analysis by the PHOBOS collaboration have shown that the fraction of the participant nucleons in the collision, $N_{\text {part }} / 2 A$ (where $A$ is the mass number) is a good scaling variable when comparing $\mathrm{Cu}+\mathrm{Cu}$ and $\mathrm{Au}+\mathrm{Au}$ collisions. The centrality evolution of $d N / d \eta$ distributions can be quantified by the $R_{P C}^{N \text { part }}$ ratio - the ratio of peripheral and central data, normalized by the number of participant nucleons, $N_{\text {part }}$ - with great experimental precision, due to the cancellation of certain systematic errors. It was found that this ratio is the same within the small uncertainties for $\mathrm{Cu}+\mathrm{Cu}$ and $\mathrm{Au}+\mathrm{Au}$ collisions, if the selected centrality bins for the two systems have the same $N_{\text {part }} / 2 A$ ratio [30]. It seems that the fraction of participating nucleons determines the evolution of the $d N / d \eta$ shape with centrality, independently of $A$. This is illustrated on Fig. 5, at the lowest RHIC energy (for that allows the largest forward coverage of the detectors relative to the beam rapidity).

In summary, a lot of simplicity and order can be found in the experimental data on the bulk features of the heavy ion collisions, which can prove useful, if not essential, in evaluating more 


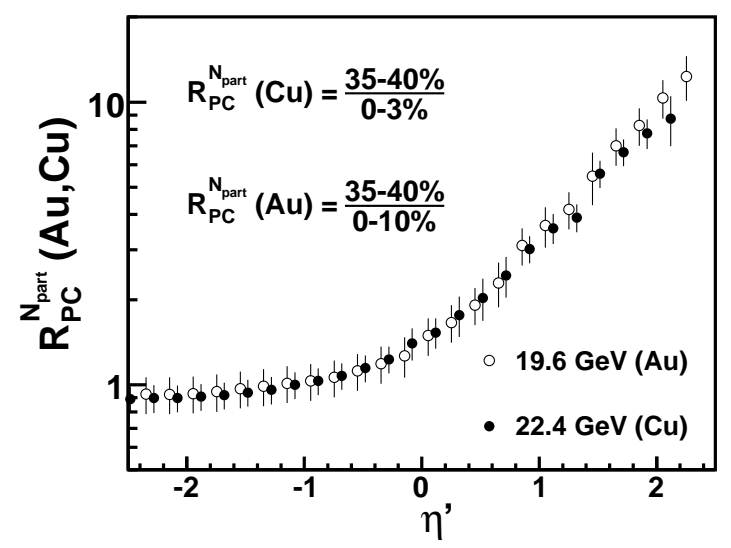

Figure 5: The ratio $R_{P C}^{N p a r t}\left(\eta^{\prime}\right)$ of the $\left(d N_{c h} / d \eta^{\prime}\right) /\left\langle N_{\text {part }}\right\rangle$ distributions for $\mathrm{Cu}+\mathrm{Cu}$ and $\mathrm{Au}+\mathrm{Au}$ collisions at the lowest RHIC energies, measured by PHOBOS in $\mathrm{Cu}+\mathrm{Cu}$ and $\mathrm{Au}+\mathrm{Au}$ collisions for centrality bins selected such that $N_{\text {part }} / 2 A$ is similar for the two systems [30]. The error bars represent a $90 \%$ C.L. systematic error on the ratio.

complicated correlation measurements. While correlations involving high- $p_{T}$ particles are intended to probe the created medium, a lot of other quantities $\left(d N / d \eta, v_{2}\right.$, event plane, centrality, system size) are necessary to characterize the event that the high- $p_{T}$ probe was embedded in.

\section{4. $d N / d \eta$ and correlation measurements with CMS}

The present, last section is devoted to preparations within the CMS heavy ion program for the measurement of particle distributions and certain correlations in the first $\mathrm{Pb}+\mathrm{Pb}$, and in the preceding first $\mathrm{p}+\mathrm{p}$ data taking run.

The silicon pixel detector is a multiple-layer, highly segmented tracking device in the proximity of the interaction point at the center of the CMS apparatus. The occupancy of the pixels will not exceed a few percent even in the $\mathrm{Pb}+\mathrm{Pb}$ data taking mode. A simple cluster counting method was developed that can be used to measure the $d N / d \eta$ distribution of charged particles, including the low- $p_{T}$ region down to $p_{T} \approx 30 \mathrm{MeV}$ [31]. Neither precise alignment, nor tracking is necessary to complete this analysis. One important component of the background is the presence of looper particles in the magnetic field that cross the pixel barrel layers multiple times. These can be discriminated against at high $\eta$ using a cut on the energy loss or the cluster width expected for particles crossing the layer at correspondingly shallow angles. The reconstructed $d N / d \eta$ distribution from the three pixel layers, together with the simulated truth, is plotted on the left panel of Fig. 6 , for $\mathrm{p}+\mathrm{p}$ collisions at $\sqrt{s}=14 \mathrm{TeV}$. Since the various types of $\mathrm{p}+\mathrm{p}$ events (single- and doublediffractive, non-diffractive etc.) contribute to the inclusive multiplicity distributions differently, the shape of the $d N / d \eta$ distribution depends on the total multiplicity. The effect can also be studied with the cluster counting method, and the reconstructed distributions together with the simulated truth in various multiplicity bins can be seen in Fig. 6 [31].

Significant effort was spent on the extension of the charged particle tracking in CMS into the low transverse momentum region, at the same time, maintaining a low fake rate both in $p+p$ and $\mathrm{Pb}+\mathrm{Pb}$ collisions. Based on the careful optimization of the specific energy loss measurement in the 

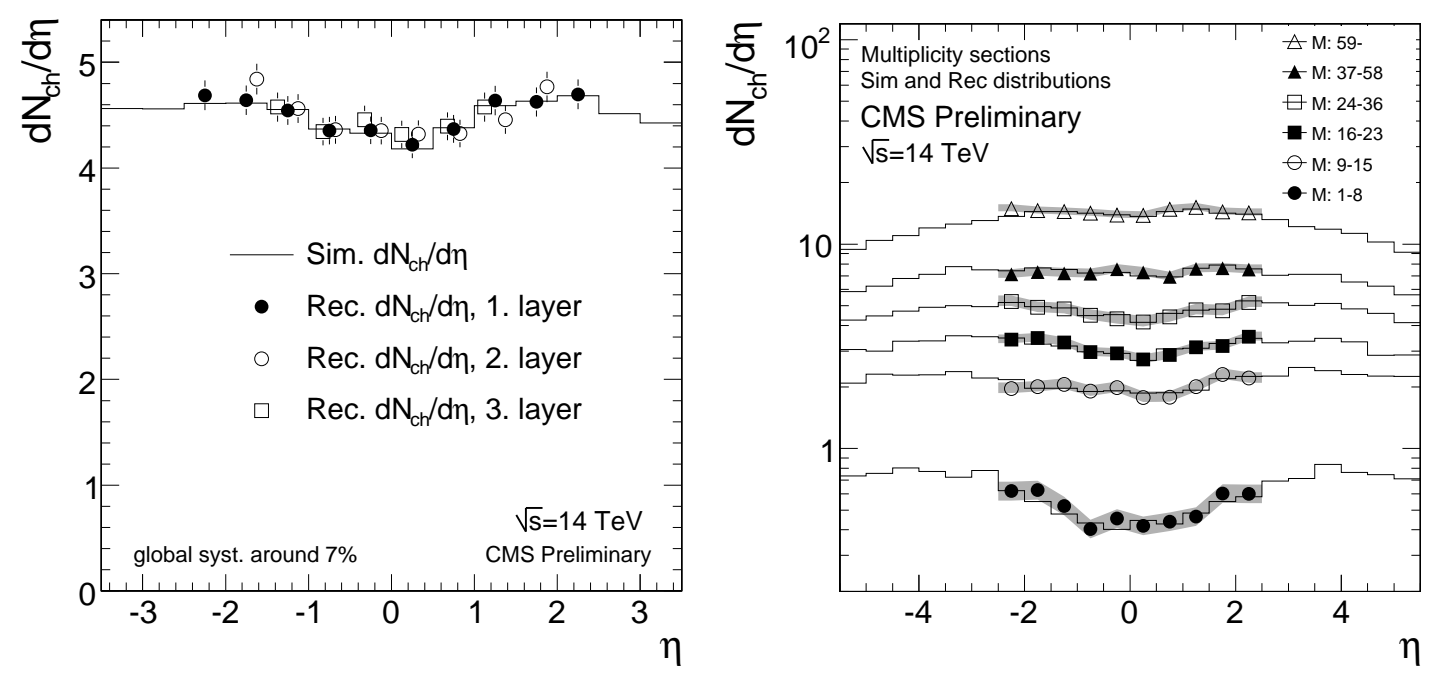

Figure 6: Left: charged particle $d N_{c h} / d \eta$ distributions of simulated (histogram) and reconstructed (symbols) $\mathrm{p}+\mathrm{p}$ events [31] at $\sqrt{s}=14 \mathrm{TeV}$ using the CMS pixel detectors. Different symbols (shifted slightly in $\eta$ for clarity) correspond to each of the three pixel layers in CMS. Error bars show the statistical errors corresponding to $5000 \mathrm{p}+\mathrm{p}$ events. Right: the same $d N_{c h} / d \eta$ distributions in six multiplicity classes, in the first CMS pixel layer. The error bands represent uncertainties from the vertex bias and from the model dependence of the corrections.

CMS silicon tracker system, the charged pions, kaons and protons can be also identified in the low momentum range [32], as illustrated in Fig. 7 together with empirical fits. The particle identification combined with a low fake rate, low- $p_{T}$ tracking opens the way to correlation measurements such as HBT correlations, other two-particle correlations, even with identified particles, resonance reconstruction, etc. The CMS tracker is covering full $2 \pi$ azimuthal angle, thus can be used to determine the event plane and the elliptic flow in $\mathrm{Pb}+\mathrm{Pb}$ collisions with high accuracy. Corresponding studies can be found in [33].

Of course, CMS is designed for reconstruction of high- $p_{T}$ objects, and it has excellent capabilities in high- $p_{T}$ tracking and jet reconstruction. Both features are utilized in studies of the nuclear modification factors, $R_{A A}$, at very high $p_{T}$ in heavy ion collisions. At the nominal $\mathrm{Pb}+\mathrm{Pb}$ luminosity at the LHC with about one month of data taking, the $R_{A A}$ ratio can be measured only up to $p_{T} \approx 100 \mathrm{GeV} / \mathrm{c}$ if the available bandwidth is filled with minimum bias events. However, the $p_{T}$ reach of the measurement can be extended to about $300 \mathrm{GeV} / \mathrm{c}$ when using a jet trigger [33], since those high- $p_{T}$ particles are originating from jet fragmentation. The expected $R_{A A}$ factors are shown in Fig. 8 without (left) and with (right) applying the jet triggers. The large coverage of the CMS calorimeters makes it possible to develop an effective and meaningful jet trigger with multiple thresholds at the nominal $\mathrm{Pb}+\mathrm{Pb}$ luminosity. The charged particle $p_{T}$ spectra from the events satisfying the trigger conditions with various thresholds are then merged together, using the minimum bias data sample.

There are several different ways to experimentally assess the parton energy loss due to the interaction with the created medium. It is expected that the collisional and radiative energy loss of the partons will modify their fragmentation functions depending on their path length travelled in 

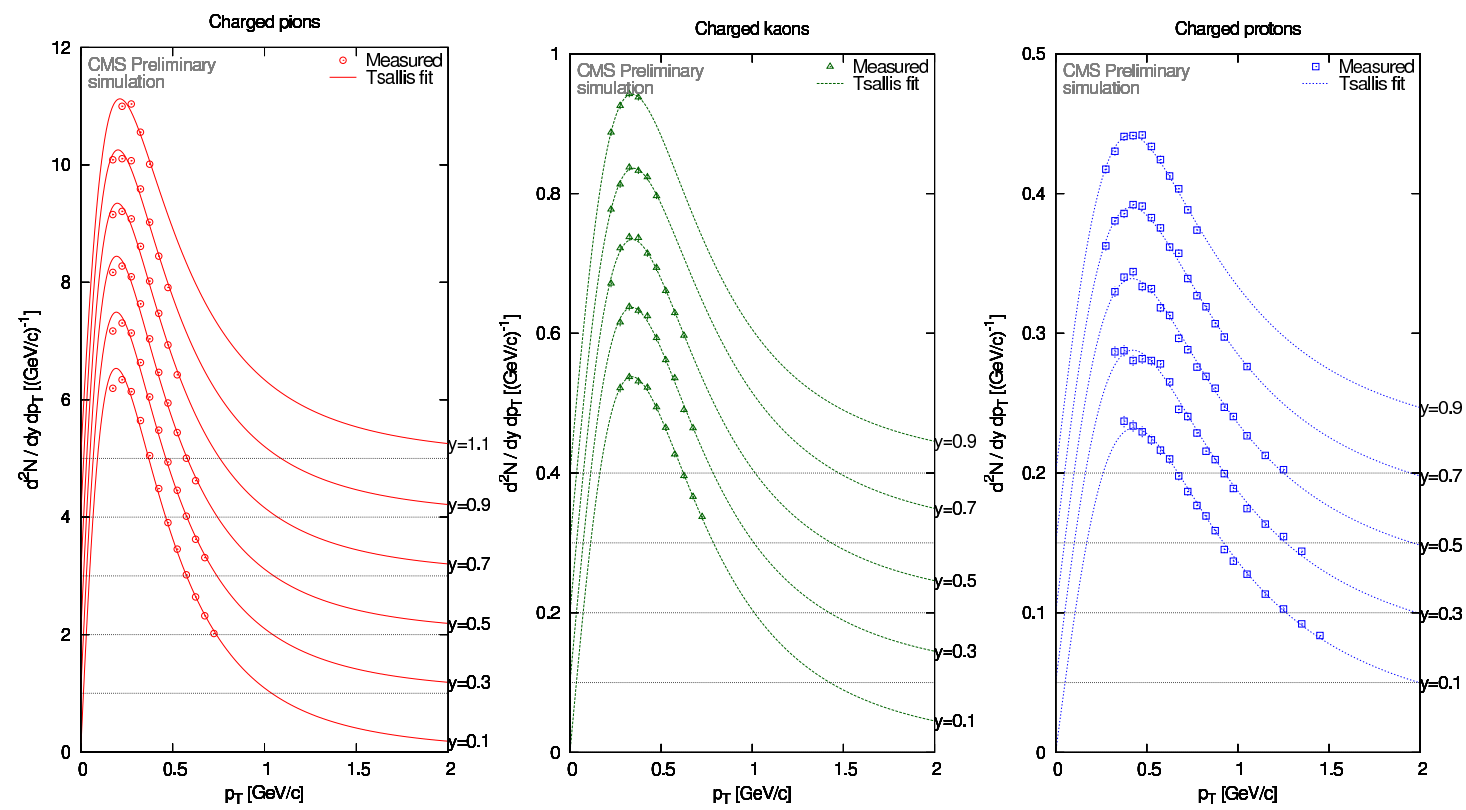

Figure 7: Differential yields of identified charged pions (left), kaons (center) and protons (right) from simulated $\mathrm{p}+\mathrm{p}$ events at $\sqrt{s}=14 \mathrm{TeV}$, using the specific energy loss in the CMS tracker, in the $0<|y|<1$ range, split into 0.2 unit wide rapidity bins, with empirical fit functions [32]. Values are successively shifted upwards by 1 for pions, by 0.1 for kaons, and by 0.05 for protons.

the medium, and on the medium density. With $\mathrm{Pb}+\mathrm{Pb}$ collisions at $\mathrm{LHC}$ energies it will be possible to perform the first detailed measurements of the in-medium modifications of jet fragmentation functions, using the $\gamma$-jet channel. Since the photon does not strongly interact with the medium, the initial $E_{T}$ of the fragmenting parton is strongly correlated to the photon $E_{T}$ in back-to-back $\gamma$-jet events. This in turn allows for precision studies of the jet fragmentation function, exploiting the CMS track reconstruction capabilities and the large rapidity coverage of the CMS calorimeters [34]. The jet reconstruction was performed with an iterative cone algorithm with a cone size of $\mathrm{R}=0.5$ modified to subtract the underlying soft background on an event-by-event basis [35].

For each photon candidate in the CMS electromagnetic calorimeter, various shape variables are examined, and together with the information from the hadronic calorimeters and the Silicon Tracker, it is determined if a given photon candidate is an isolated photon. The total transverse energy in a cone around the photon candidate, as well as the maximal hadron $p_{T}$ in the cone is limited for a photon candidate to be qualified as isolated. The final cuts are chosen to provide $60 \%$ signal efficiency for isolated photons and $95 \%$ background rejection (caused by hadrons and meson decays to $\gamma$-s), with a signal-to-background ratio of 4.5 in central $\mathrm{Pb}+\mathrm{Pb}$ collisions.

The fragmentation functions are evaluated using the reconstructed charged particles lying within a 0.5 radius cone around the jet axis on the opposite side of the isolated photon. The heavy ion background (contribution from soft particles) is subtracted using the momentum distributions of the tracks found outside the $\eta-\phi$ region occupied by the jet. An example for such a reconstructed fragmentation function from the full simulation of the CMS signal responses is shown on the left panel of Fig. 9 for non-quenched simulated $\mathrm{Pb}+\mathrm{Pb}$ events at $\sqrt{s_{N N}}=5.5 \mathrm{TeV}$. Using the HYDJET event generator [36], quenched $\mathrm{Pb}+\mathrm{Pb}$ events can be simulated as well, and the ratio of the frag- 

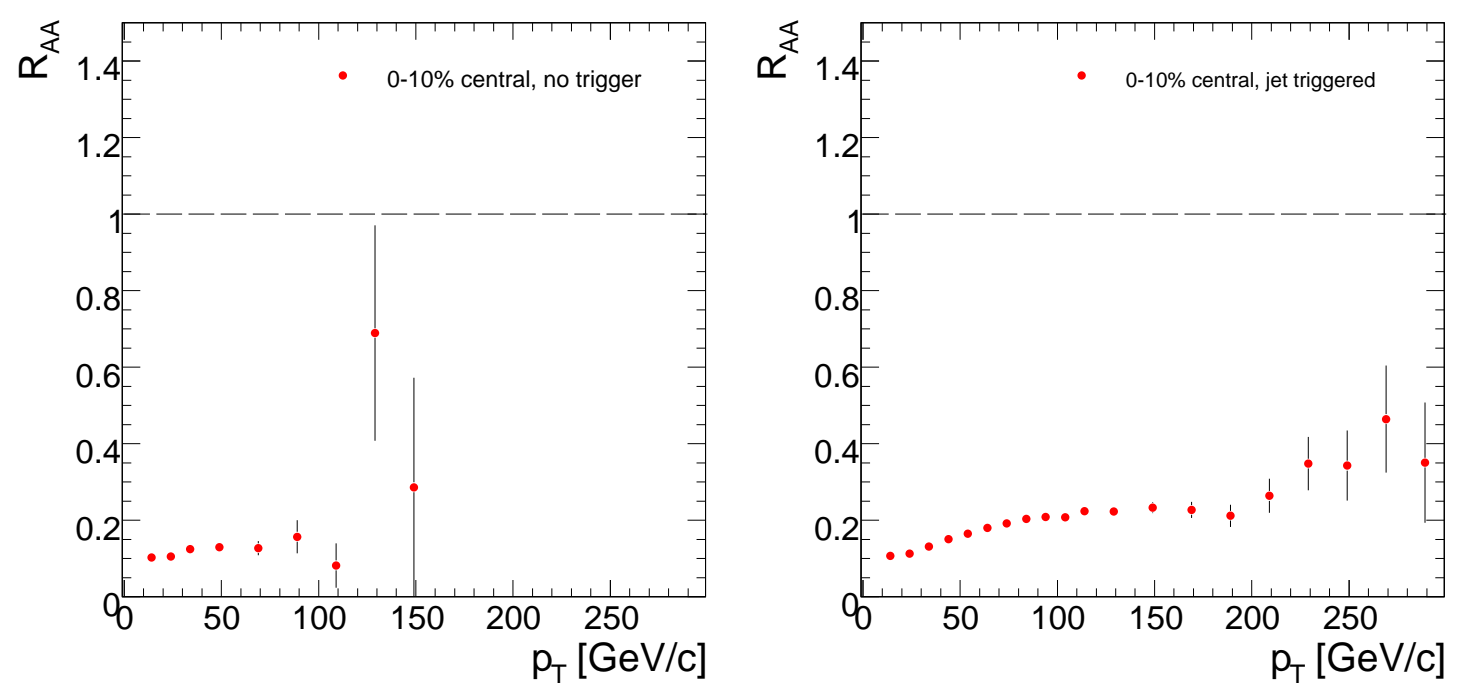

Figure 8: Expected statistical reach in the CMS experiment for the nuclear modification factor, $R_{A A}\left(p_{T}\right)$, for inclusive charged hadrons in central $\mathrm{Pb}+\mathrm{Pb}$ collisions generated with HYDJET [36] for a nominal integrated luminosity of $0.5 n b^{-1}$, for minimum bias data (left) and for data triggered on high- $E_{T}$ jets (right) [33].

mentation functions can be evaluated, shown on the right panel of Fig. 9 [34]. The deviation of this ratio from unity is quantitatively related to the parton energy loss in the created medium. Thus, for a data set corresponding to one nominal year of $\mathrm{CMS} \mathrm{Pb}+\mathrm{Pb}$ running, these measurements will be sensitive to the foreseeable changes in the fragmentation functions relative to parton fragmentation in vacuum, with the presently expected systematic and statistical uncertainties.

\section{Summary}

Correlation and multiplicity measurements in heavy ion collisions were discussed starting from the recent interest and results on correlations with a high- $p_{T}$ trigger particle by the PHOBOS experiment, that has extended the longitudinal $(\Delta \eta)$ reach of the measurement leading to consequences on the interpretation of the 'ridge' effect. The necessity of the large experimental $\eta$ coverage and the importance of the bulk properties and underlying correlations, like $d N / d \eta$ and $v_{2}$, was emphasized. Examples of spectacular scaling features of bulk quantities explored at RHIC were also presented. Finally, preparations for the LHC data taking, particularly the analysis of simulated $\mathrm{p}+\mathrm{p}$ and $\mathrm{Pb}+\mathrm{Pb}$ events were summarized, including a method to evaluate $d N / d \eta$ distributions using the early $\mathrm{p}+\mathrm{p}$ data, as well as $R_{A A}$ and jet fragmentation function measurements using triggering on jets and gamma-jet events, respectively.

\section{Acknowledgments}

The author is the member of the PHOBOS and the CMS Collaborations, from where the presented publicly available data and information were obtained. The author acknowledges the support of the Hungarian OTKA grants F 49823, T 48898, NKTH-OTKA grant H07-C 74248 and the Zoltán Magyary Postdoctoral Fellowship. 

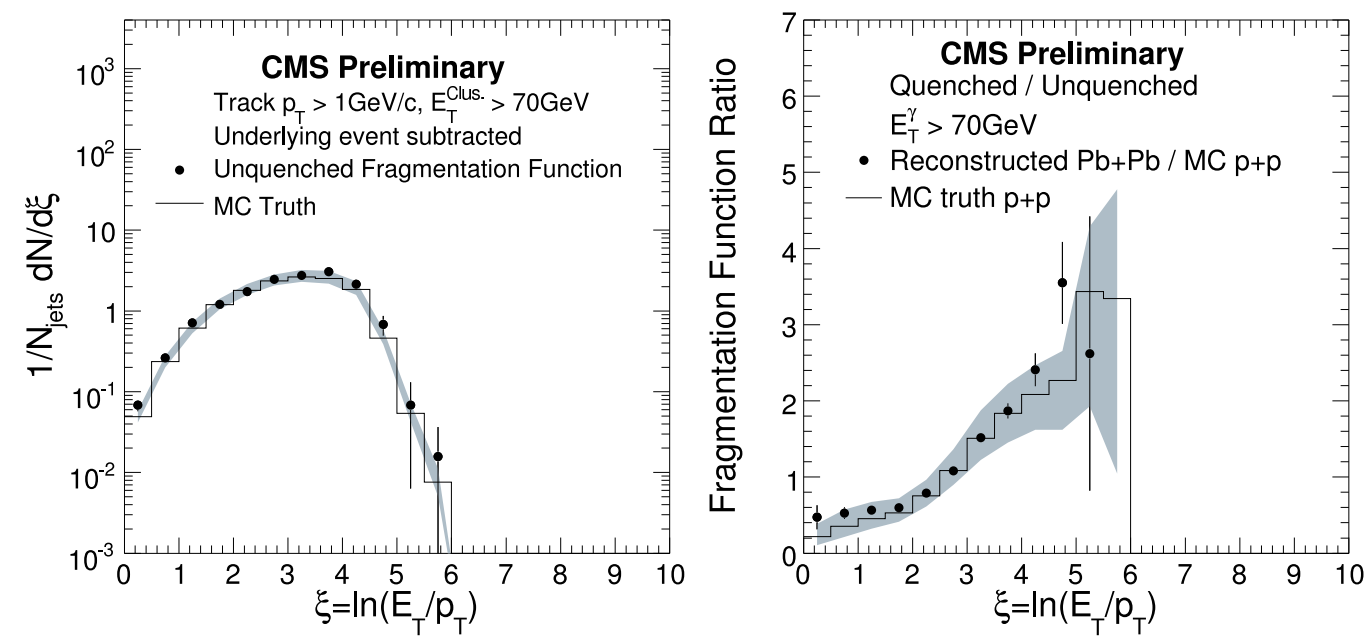

Figure 9: Left: fragmentation function extracted from simulated central $\mathrm{Pb}+\mathrm{Pb}$ collisions (symbols) and the MC truth signal (line) using the CMS apparatus at $\sqrt{s}=5.5 \mathrm{TeV} /$ nucleon pair [34]. These functions are obtained using a CMS ECAL cluster cut of $E_{T}>70 \mathrm{GeV}$ and are corrected for the underlying $\mathrm{Pb}+\mathrm{Pb}$ event. The unquenched jets were generated with PYTHIA [12], and the unquenched underlying event, generated by HYDJET [36], is subtracted. Right: ratio of the quenched to unquenched fragmentation functions for $\mathrm{Pb}+\mathrm{Pb}$ events using a minimum photon $E_{T}$ of $70 \mathrm{GeV}$. Symbols represent the ratio of the functions reconstructed from the simulated events, while the line represents the ratio of the fragmentation functions from the Monte Carlo truth.

\section{References}

[1] Adare A et al. [PHENIX] Phys. Rev. C 77, (2008) 011901(R)

[2] Adams J et al. [STAR] Phys. Rev. Lett. 95, (2005) 152301

[3] Alver B et al. [PHOBOS] J. Phys. G 35, (2008) 104080

[4] Alver B et al. [PHOBOS] Phys. Rev. Lett. (submitted), Preprint arXiv:0903.2811

[5] Wong C Y Preprint ArXiv:0712.3282 (2007)

[6] Wong C Y Phys. Rev. C 76, (2007) 054908

[7] Back B B et al. [PHOBOS] Nucl. Phys. A 757, (2005) 28

[8] Back B B et al. [PHOBOS] Phys. Lett. B578, (2004) 297

[9] Back B B et al. [PHOBOS] Phys. Rev. Lett. 91, (2003) 072302

[10] Adler S S et al. [PHENIX] Phys. Rev. C 69, (2004) 034910

[11] Adams J et al. [STAR] Phys. Rev. Lett. 91, (2003) 172302

[12] Sjöstrand T et al. Computer Phys. Commun. 135, (2001) 238, PYTHIA 6.325, default settings.

[13] Adler C et al. [STAR] Phys. Rev. Lett. 90, (2003) 082302

[14] Putschke J [STAR] J. Phys. G: Nucl. Part. Phys. 34, (2007) S679

[15] Armesto N, Salgado C and Wiedemann U Phys. Rev. Lett. 93, (2004) 242301

[16] Chiu C B and Hwa R C Phys. Rev. C 72, (2005) 034903 
[17] Romatschke P Phys. Rev. C 75, (2007) 014901

[18] Majumder A, Müller B and Bass S A Phys. Rev. Lett. 99, (2007) 042301

[19] Shuryak E V Phys. Rev. C 76, (2007) 047901

[20] Pantuev V S Preprint arXiv:0710.1882 (2007)

[21] Dumitru A, Gelis F, McLerran L, Venugopalan R Nucl. Phys. A 810, (2008) 91

[22] Back B B et al. [PHOBOS] Phys. Rev. C 72, (2005) 05190

[23] Ajitanand N N et al. Phys. Rev. C 72, (2005) 011902(R)

[24] Back B B et al. [PHOBOS] Phys. Rev. Lett. 91, (2003) 052303

[25] Veres G I [PHOBOS] Proc. of Sci. HIGH-pTLHC-017 (2009)

[26] Back B B et al. [PHOBOS] Nucl. Inst. Meth. A 499, (2003) 603

[27] Busza W Acta Phys. Polon. B 35, (2004) 2873

[28] Veres G I Nucl. Phys. A 774, (2006) 287

[29] Back B B et al. [PHOBOS] Phys. Rev. Lett. 94, (2005) 122303

[30] Alver B et al. [PHOBOS] Phys. Rev. Lett. accepted (2009) Preprint arXiv:0709.4008

[31] CMS Collaboration, CMS Physics Analysis Summary, CMS PAS QCD-08-004, https://twiki.cern.ch/twiki/bin/view/CMS/PhysicsResults

[32] CMS Collaboration, CMS Physics Analysis Summary, CMS PAS QCD-07-001, https://twiki.cern.ch/twiki/bin/view/CMS/PhysicsResults

[33] d'Enterria D et al. [CMS] J. Phys. G 34, (2007) 2307

[34] CMS Collaboration, CMS Physics Analysis Summary, CMS PAS HIN-07-002, https://twiki.cern.ch/twiki/bin/view/CMS/PhysicsResults

[35] Baur G et al. [CMS] Eur. Phys. J. C 32s02, (2004) 69

[36] Lokhtin I P and Snigirev A M Eur. Phys. J. C 46, (2006) 211 